\title{
Adaptive Multiple-Beamformers for Reception of Coherent Signals with Known Directions in the Presence of Uncorrelated Interferences
}

\author{
Linrang Zhang ${ }^{1}$, H.C. So ${ }^{2}$, Li Ping ${ }^{3}$ and Guisheng Liao ${ }^{1}$ \\ ${ }^{1}$ National Key Laboratory of Radar Signal Processing \\ Xidian University, Xi’an, China \\ ${ }^{2}$ Department of Computer Engineering \& Information Technology \\ City University of Hong Kong, Kowloon, Hong Kong \\ ${ }^{3}$ Department of Electronic Engineering \\ City University of Hong Kong, Kowloon, Hong Kong
}

\begin{abstract}
Two novel adaptive multiple-beamformers for reception of coherent signals with known directions-of-arrival (DOAs) in the presence of uncorrelated interferences are proposed. The first approach is a two-step solution as follows: estimate the amplitudes of all the coherent signals using a subspace method and then construct the linearly constrained minimum variance beamformer from the generalized array manifold. The second approach applies multiple linear constraints determined from the DOAs of the coherent signals to develop a minimum variance beamformer, which can achieve efficient signal utilization. To cope with performance degradation due to sample covariance errors, DOA estimation errors and other array imperfections, the eigenstructure of the covariance matrix is exploited to improve the performance of the proposed approaches by constraining the weight vector in the signal subspace. Simulation results are presented which compare the performance of the proposed algorithms with that of the minimum mean square error approach.
\end{abstract}

Keywords: Array processing; Blind beamforming; Coherent signals 


\section{Introduction}

Coherent sources are common in many practical problems. A well-known example is multipath propagation. Signals traveling along different paths can be considered as coherent with the original source signal if their relative delays are much smaller than the reciprocal of the signal bandwidth. Conventional adaptive beamforming approaches, which assume uncorrelated signal sources, suffer from signal cancellation in the presence of coherent signals. To eliminate signal cancellation, possible solutions [1][4] include the use of averaging over either spatial or frequency domain to destroy the coherent components prior to beamforming. After de-correlating the coherent signals, the beamformer can then put nulls in their directions-of-arrival (DOAs). This approach is further refined in [5] by the use of high-order null constraints in the DOAs associated with the coherent signals. In [6,7], signal cancellation in the presence of coherent interferences is attributed to the non-Toeplitz structure of the estimated covariance matrix and techniques of redundancy averaging and enhanced redundancy averaging are proposed, which put nulls in the DOAs of the coherent interferences.

However, in some scenarios, such as multipath propagation, steering the nulls in the directions of the coherent signals is not desirable. This is because to fully exploit the information of the coherent signals a beamformer should constructively combine these signals instead of canceling all but one of them. A cumulant-based blind beamformer has been developed in [8] in the presence of coherent multipath propagation. In this approach, multipath components are utilized instead of de-correlated, but this is only feasible in situations where the desired signal and the interference are non-Gaussian and Gaussian, respectively. A two-stage procedure for the reception of coherent signals and the suppression of strong interference has also been proposed in [9]. The first step uses an interference-blocking (IB) transformation to remove the strong interference, while retaining the desired signals and noise. Optimum beamforming is then performed based on the IB transformation data to maximize the output signal-to-interference-plus-noise ratio (SINR). However, this method is only suitable for a very small signal-tointerference ratio. By constructing a steering matrix with each column corresponding to the steering vector of a selective beam and a constraint vector with each entry equal to the gain of a beam, [10] proposes an adaptive array beamformer with multiple-beam 
constraints, although the details of obtaining the constraint vector are not discussed. A beamforming scheme, consisting of three steps, is developed for the reception of multiple coherent signals in [11]. Here, estimates of the coherent source directions are used to restore the composite steering vector (CSV). A transformation is then carried out to remove the coherent signals while retaining the interference and noise. Finally, optimum beamforming is performed based on the CSV and the transformed data to maximize the output SINR. However, this scheme needs the DOA information of all signals, including that of the interferences.

Coherent signal beamforming can be achieved by using an antenna array with a multiple-beam pattern selected such that the beams are directed to all the coherent signals and their gains are proportional to the corresponding coherent signal amplitudes. In this paper, we present two adaptive beamforming approaches which use multiple beams for coherent signal reception as well as interference suppression. We assume that the DOA information of the coherent signals is available or has already been estimated [12]-[15] but their amplitudes are not known a priori.

The rest of the paper is organized as follows. The data model is presented in Section II. Two new beamforming approaches, the first a linearly constrained minimum variance (LCMV) beamformer and the second a multiple constrained minimum variance (MCMV) beamformer, are developed in Section III. Section III also reviews the optimum minimum mean square error (MMSE) combiner and statistically optimal interferenceplus-noise rejecter. The performance of the proposed LCMV and MCMV beamformers is analyzed in Section IV. To overcome the problem arising from sample covariance errors due to finite samples, DOA estimation errors and other array imperfections, the use of an eigenspace-based beamformer that constrains the weight vector in the signal subspace is also discussed in Section IV. In Section V, simulation results are presented to illustrate the effectiveness of the proposed methods. Finally, conclusions are drawn in Section VI.

\section{Data Model}

Assume that plane waves emitted by a group of $P$ coherent sources and $J$ uncorrelated interferences impinge on a $M$-element antenna array from directions $\theta_{d_{i}}$, $i=1,2, \ldots, P$ and $\theta_{u_{i}}, i=1,2, \ldots, J$, respectively, where the numbers of the coherent signals 
and interference sources and the bearings of the coherent sources are known a priori. All the sources are assumed to be narrowband with the same center frequency and in the far field of the array. The received array signal vector can be expressed as

$$
\mathbf{x}(t)=s_{d}(t) \sum_{i=1}^{P} \alpha_{i} \mathbf{a}\left(\theta_{d_{i}}\right)+\sum_{i=1}^{J} s_{i}(t) \mathbf{a}\left(\theta_{u_{i}}\right)+\mathbf{n}(t)
$$

where $s_{d}(t)$ represents the desired signal waveform, the quantity $\alpha_{i}$ represents the complex amplitude of the $i$ th coherent signal and $\left|\alpha_{i}\right|<1, i=1, \ldots, P$. The $M \times 1$ vector $\mathbf{a}(\theta)$ is called the steering vector of the array, and $s_{i}(t)$ represents the $i$ th interference with power $\sigma_{i}^{2}=E\left\{\left|s_{i}(t)\right|^{2}\right\}, i=1,2, \ldots, J$. The $\mathbf{n}(t)$ is a spatially white noise vector with power $\sigma_{n}{ }^{2}$. It is assumed that $s_{d}(t),\left\{s_{i}(t)\right\}$, and $\mathbf{n}(t)$ are uncorrelated with each other. For a more general data model, interested readers can refer to [7]. The signal-to-noise ratio (SNR) for the $i$ th coherent signal is defined as $S N R_{i}=\frac{E\left\{\left|\alpha_{i} \cdot s_{d}(t)\right|^{2}\right\}}{\sigma_{n}{ }^{2}}$, and interference-to-noise ratio (INR) for the ith interference is defined as $I N R_{i}=\frac{\sigma_{i}^{2}}{\sigma_{n}{ }^{2}}$, respectively. Denote the composite vector associated with the coherent sources by

$$
\tilde{\mathbf{a}}=\sum_{i=1}^{P} \alpha_{i} \mathbf{a}\left(\theta_{d_{i}}\right)=\mathbf{A} \cdot \boldsymbol{\alpha},
$$

where $\mathbf{A}=\left[\mathbf{a}\left(\theta_{d_{1}}\right), \mathbf{a}\left(\theta_{d_{2}}\right), \cdots, \mathbf{a}\left(\theta_{d_{P}}\right)\right]$ and $\boldsymbol{\alpha}=\left[\alpha_{1}, \alpha_{2}, \cdots, \alpha_{P}\right]^{T}$. Note that $\widetilde{\mathbf{a}}$ is also referred to as the generalized array manifold (GAM) [8] of the desired signal. If we further denote the interference vector by $\mathbf{i}(t)=\sum_{i=1}^{J} s_{i}(t) \mathbf{a}\left(\theta_{u_{i}}\right)$, then (1) can be written as

$$
\mathbf{x}(t)=s_{d}(t) \tilde{\mathbf{a}}+\mathbf{i}(t)+\mathbf{n}(t) .
$$

The covariance matrix of $\mathbf{x}(t)$ is given by $\mathbf{R} x=E\left\{\mathbf{x}(t) \mathbf{x}(t)^{H}\right\}$, where $(.)^{\mathrm{H}}$ denotes conjugate transposition. Given $\mathbf{x}(t)$ and $\mathbf{A}$, the aim is to devise optimum beamforming weight vectors. 


\section{Development of New Beamformers}

The MMSE combiner is first reviewed. If the waveform of the desired signal is known, that is when a training signal is available, the optimum weight vector $\mathbf{w}$ for processing $\mathbf{x}(t)$ is computed by minimizing the following mean square error function:

$$
E\left\{\left|S_{d}(t)-\mathbf{w}^{H} \cdot \mathbf{x}(t)\right|^{2}\right\} .
$$

The MMSE solution is given by [2]

$$
\mathbf{w}_{\mathrm{MMSE}}=\mathbf{R}_{x x}{ }^{-1} \mathbf{r}_{x s},
$$

where $\mathbf{r}_{x s}=E\left\{\mathbf{x}(t) \cdot s_{d}^{*}(t)\right\},(.)^{*}$ is the conjugate operation.

Another well known beamformer is the statistically optimal interference-plusnoise rejecter [2], whose weight vector is chosen to pass the desired signal to the beamformer output while maximally rejecting the contribution of the interferences and noise. The corresponding weight vector is determined as

$$
\mathbf{w}_{\text {opt }}=\arg \min _{\mathbf{w}} \mathbf{w}^{H} \mathbf{R}_{\text {in }} \mathbf{w}, \quad \text { subject to } \mathbf{w}^{H} \tilde{\mathbf{a}}=1,
$$

where $\quad \mathbf{R}_{\text {in }}$ is the interference-plus-noise covariance or signal-free covariance. The solution to (6) is

$$
\mathbf{W}_{\mathrm{opt}}=\mathbf{R}_{i n}^{-1} \cdot \tilde{\mathbf{a}} /\left(\tilde{\mathbf{a}}^{H} \cdot \mathbf{R}_{i n}{ }^{-1} \cdot \tilde{\mathbf{a}}\right) .
$$

Note that $\mathbf{r}_{x s}=\sigma_{d}{ }^{2} \tilde{\mathbf{a}}$ and $\mathbf{R}_{x x}=\mathbf{R}_{i n}+\sigma_{d}{ }^{2} \tilde{\mathbf{a}} \tilde{\mathbf{a}}^{H}$ in (5). Using the matrix inversion lemma, we have

$$
\left(\mathbf{R}_{i n}+\sigma_{d}{ }^{2} \tilde{\mathbf{a}} \tilde{\mathbf{a}}^{H}\right)^{-1}=\mathbf{R}_{i n}{ }^{-1}-\frac{\sigma_{d}{ }^{2}}{1+\sigma_{d}{ }^{2} \tilde{\mathbf{a}}^{H} \cdot \mathbf{R}_{i n}{ }^{-1} \cdot \tilde{\mathbf{a}}} \mathbf{R}_{i n}{ }^{-1} \tilde{\mathbf{a}} \tilde{\mathbf{a}}^{H} \mathbf{R}_{i n}{ }^{-1},
$$

and with the use of (7), (5) can be expressed as

$$
\mathbf{W}_{\mathrm{MMSE}}=\frac{\sigma_{d}{ }^{4} \tilde{\mathbf{a}}^{H} \mathbf{R}_{i n}{ }^{-1} \tilde{\mathbf{a}}}{1+\sigma_{d}{ }^{2} \tilde{\mathbf{a}}^{H} \cdot \mathbf{R}_{i n}{ }^{-1} \cdot \tilde{\mathbf{a}}} \mathbf{w}_{o p t} .
$$

Since $\tilde{\mathbf{a}}^{H} \mathbf{R}_{\text {in }}{ }^{-1} \tilde{\mathbf{a}}$ is a scalar, (8) indicates that the MMSE combiner is equivalent to the statistically optimal interference-plus-noise rejecter.

In the MMSE beamformer and the statistically optimal interference-plus-noise rejecter, the multiple correlated waveforms corresponding to the coherent signals are optimally combined to maximize the output SINR. However, the use of a training signal 
implies a waste of bandwidth and a training signal may not be available in many practical situations, and the interference-plus-noise covariance cannot obtained directly from the received data. In the following sections, two blind beamforming approaches, which require no training signals, are proposed.

\section{A. LCMV Beamformer based on GAM estimation (LCMV-GAM)}

If the GAM of the desired signal is known, that is the DOAs and complex amplitudes of the coherent signals are available, a standard beamforming strategy is to use the LCMV criterion, which chooses the weight vector as

$$
\left.\mathbf{w}_{\text {LCMV-GAM }}=\left.\arg \min _{\mathbf{w}} E\left\{\mid \mathbf{w}^{H} \cdot \mathbf{x}(t)\right)\right|^{2}\right\}, \quad \text { subject to } \tilde{\mathbf{a}}^{H} \mathbf{w}=1 .
$$

The LCMV-GAM beamformer is given by

$$
\mathbf{W}_{\text {LCMV-GAM }}=\frac{\mathbf{R}_{x x}^{-1} \cdot \tilde{\mathbf{a}}}{\tilde{\mathbf{a}}^{H} \cdot \mathbf{R}_{x x^{-1}} \cdot \tilde{\mathbf{a}}} .
$$

Since we do not have complete knowledge of $\widetilde{\mathbf{a}}$, the unknown amplitude parameters, $\boldsymbol{\alpha}$, are first estimated prior to computing (10). Orthogonality between the noise and signalplus-interference subspaces is exploited to determine $\boldsymbol{\alpha}$ using a two-step procedure:

Step 1. Identify the signal-plus-interference subspace from the eigen-decomposition of the covariance matrix $\mathbf{R}_{x x}$ :

$$
\mathbf{R}_{x x}=E\left\{\mathbf{x}(t) \mathbf{x}(t)^{H}\right\}=\sum_{i=1}^{M} \lambda_{i} \mathbf{e}_{i} \mathbf{e}_{i}{ }^{H}=\mathbf{E}_{s} \boldsymbol{\Lambda}_{s} \mathbf{E}_{s}{ }^{H}+\mathbf{E}_{n} \boldsymbol{\Lambda}_{n} \mathbf{E}_{n}{ }^{H},
$$

where $\lambda_{1} \geq \lambda_{2} \geq \cdots \geq \lambda_{J+1} \geq \lambda_{J+2}=\cdots=\lambda_{M}=\sigma_{n}{ }^{2}$ are the eigenvalues, and $\mathbf{e}_{\mathbf{i}}, \mathrm{i}=1,2, \ldots$, $M$, are the corresponding orthonormal eigenvectors. It is assumed that the vectors $\{\widetilde{\mathbf{a}}$, $\left.\mathbf{a}\left(\theta_{u_{1}}\right), \mathbf{a}\left(\theta_{u_{2}}\right), \ldots, \mathbf{a}\left(\theta_{u_{J}}\right)\right\}$ are all distinct which means that they span a subspace of dimension $J+1$. We further assume that both the SNR and INR are sufficiently large to regard the signal and interference sources as the main contributors to the largest $J+1$ eigenvalues of $\mathbf{R}_{x x}$. The signal-plus-interference subspace is then identified as that one spanned by the columns of the matrix $\mathbf{E}_{\mathrm{s}}=\left[\mathbf{e}_{1}, \mathbf{e}_{2}, \ldots, \mathbf{e}_{\mathrm{J}+1}\right]$. Similarly, the columns of the matrix $\mathbf{E}_{\mathrm{n}}=\left[\mathbf{e}_{\mathrm{J}+2}, \mathbf{e}_{\mathrm{J}+3}, \ldots, \mathbf{e}_{\mathrm{M}}\right]$ spans a subspace, identified as noise alone subspace. Any of the vectors $\left\{\widetilde{\mathbf{a}}, \mathbf{a}\left(\theta_{u_{1}}\right), \mathbf{a}\left(\theta_{u_{2}}\right), \ldots, \mathbf{a}\left(\theta_{u_{J}}\right)\right\}$ should have a minimal projection onto the noise alone subspace which accounts for Step 2 as follows, 
Step 2. Since $\widetilde{\mathbf{a}}=\mathbf{A} \cdot \boldsymbol{\alpha}$ is in the signal subspace, $\boldsymbol{\alpha}$ can be estimated from the noise subspace as

$$
\hat{\boldsymbol{\alpha}}=\arg \min _{\boldsymbol{\alpha}}\left\|\mathbf{E}_{n}{ }^{H}(\mathbf{A} \cdot \boldsymbol{\alpha})\right\|^{2}=\arg \min _{\boldsymbol{\alpha}} \boldsymbol{\alpha}^{H} \mathbf{Q} \boldsymbol{\alpha}, \quad \text { subject to }\|\boldsymbol{\alpha}\|^{2}=1,
$$

where $\mathbf{Q}=\mathbf{A}^{H} \mathbf{E}_{n} \mathbf{E}_{n}{ }^{H} \mathbf{A}$, which is equivalent to

$$
\hat{\boldsymbol{\alpha}}=\arg \min _{\boldsymbol{\alpha}}\left\{\frac{\boldsymbol{\alpha}^{H} \mathbf{Q} \boldsymbol{\alpha}}{\boldsymbol{\alpha}^{H} \boldsymbol{\alpha}}\right\} .
$$

Note that the constraint of $\|\boldsymbol{\alpha}\|^{2}=1$ is to prevent the trivial solution of zero. A necessary condition for the uniqueness of $\hat{\boldsymbol{\alpha}}$ in (13) is strict convexity of the quadratic form $\boldsymbol{\alpha}^{H} \mathbf{Q} \boldsymbol{\alpha}$ or equivalently positive definiteness of $\mathbf{Q}$ which is of dimensions $P \times P$. Hence, we should have

$$
\operatorname{rank}(\mathbf{Q})=\min \left[\operatorname{rank}(\mathbf{A}), \operatorname{rank}\left(\mathbf{E}_{n}\right)\right]=P,
$$

i.e.

$$
\min [P, M-J-1]=P,
$$

or

$$
M>1+J+P
$$

which imposes a constraint on the array length.

When $\mathbf{Q}$ is positive definite, it is well known that $\hat{\boldsymbol{\alpha}}$ is the eigenvector corresponding to the minimum eigenvalue of $\mathbf{Q}$. Substituting $\hat{\boldsymbol{\alpha}}$ into (10) yields the LCMV-GAM solution.

\section{B. MCMV Beamformer}

The motivation for the second approach is the use of the DOA information of the coherent signals to construct multiple constraints for the minimum variance beamformer, such that the coherent signals are preserved and signal cancellation is prevented. The MCMV beamformer is determined from the following constrained optimization problem $[5]$ :

$$
\mathbf{w}_{\mathrm{MCMV}}=\arg \min _{\mathbf{w}} E\left\{\left|\mathbf{w}^{H} \cdot \mathbf{x}(t)\right|^{2}\right\}, \quad \text { subject to } \mathbf{A}^{H} \mathbf{w}=\mathbf{f},
$$

where $\mathbf{f}$ is the $P \times 1$ unknown response vector which is to be determined. It is clear that $\mathbf{f}$ is dependent on $\boldsymbol{\alpha}$. According to the theory of adaptive arrays, the optimal weight vector for (17) is given by 


$$
\mathbf{w}_{\mathrm{MCMV}}=\mathbf{R} x x^{-1} \mathbf{A}\left(\mathbf{A}^{H} \mathbf{R}_{x x^{-1}} \mathbf{A}\right)^{-1} \mathbf{f} .
$$

In fact, the choice of the constrained vector $\mathbf{f}$ has a significant effect on the system performance. In [5], f is chosen as $[1,0, \ldots, 0]^{T}$, which corresponds to preserving the signal in only one desired direction and forcing the receiver response in the direction of the remaining $(P-1)$ coherent signals to zero. Although the additional constraints prevent signal cancellation due to coherent signals, this method does not make use of the energy in the coherent signal components and, therefore, is not optimal.

There have been many successful examples of cross-fertilisation between the areas of array processing and channel identification. For example, Honig et al [16] apply an idea from array processing to channel estimation via optimizing the receiver's output energy while constraining the response of the user of interest to be constant. An extension of this work to the multipath case is investigated in [17], where a max/min approach for optimizing the constraints is devised. We use the approach in [17] to obtain the constrained vector in (17) as follows.

Using (18), the array output power can be expressed as

$$
P_{\text {out }}=E\left\{\left|\mathbf{w}^{H} \cdot \mathbf{x}(t)\right|^{2}\right\}=\mathbf{f}^{H}\left(\mathbf{A}^{H} \mathbf{R}_{x x^{-1}} \mathbf{A}\right)^{-1} \mathbf{f} .
$$

After preserving the desired signals and suppressing the interference using the multiple constraints, an optimal constrained vector is obtained by maximizing (19) subject to $\|\mathbf{f}\|^{2}=1$, i.e.,

$$
\hat{\mathbf{f}}=\arg \max _{\mathbf{f}} P_{\text {out }}, \quad \text { subject to }\|\mathbf{f}\|^{2}=1 .
$$

where the constraint $\|\mathbf{f}\|^{2}=1$ accounts for the unique solution on unit circle. Equation (20) is equivalent to

$$
\hat{\mathbf{f}}=\arg \max _{\mathbf{f}} \frac{\mathbf{f}^{H}\left(\mathbf{A}^{H} \mathbf{R} x x^{-1} \mathbf{A}\right)^{-1} \mathbf{f}}{\mathbf{f}^{H} \mathbf{f}}
$$

The solution to (21) is the eigenvector corresponding to the maximum eigenvalue of $\left(\mathbf{A}^{H} \mathbf{R} x x^{-1} \mathbf{A}\right)^{-1}$. It is apparent that the maximum eigenvalue of $\left(\mathbf{A}^{H} \mathbf{R}_{x x}{ }^{-1} \mathbf{A}\right)^{-1}$ is equivalent to the minimum eigenvalue of $\left(\mathbf{A}^{H} \mathbf{R} x{ }^{-1} \mathbf{A}\right)$. Thus, (21) is equivalent to 


$$
\hat{\mathbf{f}}=\arg \min _{\mathbf{f}} \frac{\mathbf{f}^{H}\left(\mathbf{A}^{H} \mathbf{R} x x^{-1} \mathbf{A}\right) \mathbf{f}}{\mathbf{f}^{H} \mathbf{f}} .
$$

We see that (22) is more computationally efficient than (21) because no matrix inversion is required. The solution to (22) is the eigenvector corresponding to the minimum eigenvalue of $\left(\mathbf{A}^{H} \mathbf{R}_{x x}{ }^{-1} \mathbf{A}\right)$. Substituting $\hat{\mathbf{f}}$ into (18) yields the MCMV solution.

\section{Performance Analysis}

It is shown in [17] that for strong SNR and INR, the solution of (22) converges to

$$
\hat{\mathbf{f}}=\arg \min _{\mathbf{f}} \frac{\mathbf{f}^{H}\left(\mathbf{A}^{H} \mathbf{E}_{n} \mathbf{E}_{n}{ }^{H} \mathbf{A}\right) \mathbf{f}}{\mathbf{f}^{H} \mathbf{f}}
$$

Comparing (23) with (13) yields

$$
\hat{\mathbf{f}} \approx \hat{\boldsymbol{\alpha}}
$$

On the other hand, let the maximum eigenvalue of $\left(\mathbf{A}^{H} \mathbf{R} x x^{-1} \mathbf{A}\right)^{-1}$ be $\gamma_{\max }$. Then the solution of (21) satisfies

$$
\left(\mathbf{A}^{H} \mathbf{R} x x^{-1} \mathbf{A}\right)^{-1} \hat{\mathbf{f}}=\gamma_{\max } \cdot \hat{\mathbf{f}} .
$$

Substituting (25) into (18), we obtain

$$
\mathbf{w}_{\mathrm{MCMV}}=\gamma_{\max } \cdot \mathbf{R}_{x x^{-1} \mathbf{A} \cdot \hat{\mathbf{f}} .}
$$

Using (24) and comparing (26) with (10) gives

$$
\mathbf{w}_{\mathrm{MCMV}}=\mu \cdot \mathbf{w}_{\mathrm{LCMV}-\mathrm{GAM}},
$$

where $\mu=\gamma_{\max } \cdot\left(\widetilde{\mathbf{a}}^{H} \cdot \mathbf{R}_{x x^{-1}} \cdot \widetilde{\mathbf{a}}\right)$. As $\mu$ is a scalar, (27) indicates that the MCMV and LCMV-GAM beamformers are equivalent when both the SNR and INR are sufficiently large. However, when the noise is strong enough, $\hat{\boldsymbol{\alpha}}$ can be shown to be an unbiased estimate of $\alpha$ in the LCMV-GAM method, while the constrained vector $\hat{\mathbf{f}}$ is a biased estimate of $\alpha$ [17]. The bias, in terms of the vector norm, is proportional to the noise power. Consequently, it is expected that the LCMV-GAM method will outperform the MCMV beamformer at lower SNR conditions.

Furthermore, since the LCMV and MMSE beamformers for single uncorrelated source are equivalent if the DOA is known exactly, it can be deduced that the LCMV- 
GAM and MMSE beamformers for coherent sources will have identical performance when the GAM is perfectly known. This also implies that the former will degrade in comparison with the latter when the GAM is subject to estimation errors.

It is well known that calibration errors can seriously degrade performance in adaptive arrays. Similar degradation occurs when inverting the estimated covariance matrix, and the performance loss is particularly large when the input SNR is high [18-20]. To overcome these problems, [18] proposes an eigenspace-based beamformer that constrains the weight vector in the signal subspace, while [19] suggests a projection method that projects the steering vector onto the signal subspace. While the methods described in [18] and [19] are different, the weight vectors generated by them are equivalent and result in the same output SINR [20].

Apart from the impact of additive noise, imperfect estimation of the covariance matrix owing to the use of a finite number of data samples, DOA estimation errors and other array imperfections also significantly degrade the performance of the LCMV-GAM and MCMV beamformers. Here we utilize the eigenstructure of the correlation matrix to enhance their performance. The improved weight vectors are found by projecting the LCMV-GAM and MCMV weight vectors onto a subspace constructed from the eigenstructure of the correlation matrix [19]. We refer the improved methods to as E-LCMV-GAM and E-MCMV beamformers, respectively.

\section{A. Eigenspace-based LCMV-GAM Beamformer}

The LCMV-GAM beamformer weight vector can be written as

$$
\mathbf{W}_{\mathrm{LCMV}-\mathrm{GAM}}=\frac{\mathbf{R}_{x x} x^{-1} \cdot \tilde{\mathbf{a}}}{\tilde{\mathbf{a}}^{H} \cdot \mathbf{R}_{x x}{ }^{-1} \cdot \tilde{\mathbf{a}}}=\frac{1}{\tilde{\mathbf{a}}^{H} \cdot \mathbf{R}_{x x^{-1}} \cdot \tilde{\mathbf{a}}} \cdot\left[\mathbf{E}_{s} \boldsymbol{\Lambda}_{s}^{-1} \mathbf{E}_{s}{ }^{H}+\mathbf{E}_{n} \boldsymbol{\Lambda}_{n}{ }^{-1} \mathbf{E}_{n}{ }^{H}\right] \cdot \tilde{\mathbf{a}}=\mathbf{w}_{s}+\mathbf{w}_{n},
$$

where $\quad \mathbf{w}_{s}=\frac{1}{\tilde{\mathbf{a}}^{H} \cdot \mathbf{R}_{x x}{ }^{-1} \cdot \tilde{\mathbf{a}}} \cdot \mathbf{E}_{s} \boldsymbol{\Lambda}_{s}{ }^{-1} \mathbf{E}_{s}{ }^{H} \cdot \tilde{\mathbf{a}} \quad$ and $\quad \mathbf{w}_{n}=\frac{1}{\tilde{\mathbf{a}}^{H} \cdot \mathbf{R}_{x x} \cdot{ }^{-1} \cdot \tilde{\mathbf{a}}} \cdot \mathbf{E}_{n} \boldsymbol{\Lambda}_{n}{ }^{-1} \mathbf{E}_{n}{ }^{H} \cdot \tilde{\mathbf{a}}$. Ideally, $\mathbf{E}_{n}{ }^{H} \cdot \widetilde{\mathbf{a}}=0$, which means that $\mathbf{w}_{\text {LCMV-GAM }}$ is in the signal subspace or $\mathbf{w}_{n}=0$ and $\mathbf{w}_{\text {LCMV-GAM }}=\mathbf{w}_{s}$. Nevertheless, due to the effects of finite samples, pointing errors and other array imperfections, the computed $\mathbf{w}_{\text {LCMV-GAM }}$ does not remain in the signal subspace, and consequently, $\mathbf{w}_{n}$ is not equal to zero, which causes degradation of the 
output SINR. In [19], the eigenspace-based beamformer discards $\mathbf{w}_{n}$ and only uses $\mathbf{w}_{s}$ as the weight vector. As a result, the weight vector of the eigenspace-based LCMVGAM beamformer is given by

$$
\mathbf{W}_{\mathrm{E}-\mathrm{LCMV}-\mathrm{GAM}}=\frac{1}{\tilde{\mathbf{a}}^{H} \mathbf{E}_{s} \boldsymbol{\Lambda} s^{-1} \mathbf{E}_{s}^{H} \tilde{\mathbf{a}}} \mathbf{E}_{s} \boldsymbol{\Lambda} s^{-1} \mathbf{E}_{s}{ }^{H} \tilde{\mathbf{a}} .
$$

Analysis has shown [19] that the eigenspace-based beamformer has better convergence properties and is less sensitive to pointing errors than the conventional LCMV bemformer, and this is also true for the E-LCMV-GAM beamformer.

\section{B. Eigenspace-based MCMV Beamformer}

Similar to the E-LCMV-GAM beamformer, the weight vector of the eigenspacebased MCMV beamformer can be written as

$$
\begin{aligned}
\mathbf{w}_{\mathrm{E}-\mathrm{MCMV}} & =\mathbf{E}_{s} \mathbf{E}_{s}{ }^{H} \mathbf{w}_{\mathrm{MCMV}} \\
& =\mathbf{E}_{s} \mathbf{E}^{H}{ }^{H} \cdot \mathbf{R} x x^{-1} \mathbf{A}\left(\mathbf{A}^{H} \mathbf{R}_{x x^{-1}} \mathbf{A}\right)^{-1} \mathbf{f} .
\end{aligned}
$$

The output power is

$$
\begin{aligned}
P_{\text {out }} & =E\left\{\left|\mathbf{w}_{\mathrm{E}: \mathrm{McNV}}{ }^{H} \cdot \mathbf{x}(t)\right|^{2}\right\} \\
& =\mathbf{f}^{H}\left(\mathbf{A}^{H} \mathbf{R}_{x x}{ }^{-1} \mathbf{A}\right)^{-1} \mathbf{A}^{H} \mathbf{R}_{x x}{ }^{-1} \mathbf{E}_{s} \mathbf{E}_{s}{ }^{H} \mathbf{R}_{x x^{-1}} \mathbf{A}\left(\mathbf{A}^{H} \mathbf{R}_{x x}{ }^{-1} \mathbf{A}\right)^{-1} \mathbf{f}
\end{aligned}
$$

where $\mathbf{f}$ is determined from

$$
\hat{\mathbf{f}}=\arg \max _{\mathbf{f}} P_{\text {out }}, \quad \text { subject to }\|\mathbf{f}\|^{2}=1 .
$$

The solution is

$$
\hat{\mathbf{f}}=\arg \max _{\mathbf{f}} \frac{\mathbf{f}^{H} \mathbf{Q}_{s}^{-1} \mathbf{f}}{\mathbf{f}^{H} \mathbf{f}}=\arg \min _{\mathbf{f}} \frac{\mathbf{f}^{H} \mathbf{Q}_{s} \mathbf{f}}{\mathbf{f}^{H} \mathbf{f}}
$$

where

$$
\mathbf{Q}_{s}=\left(\mathbf{A}^{H} \mathbf{R}_{x x^{-1}} \mathbf{A}\right)^{-1} \mathbf{A}^{H} \mathbf{R}_{x x^{-1}} \mathbf{E}_{s} \mathbf{E}_{s}{ }^{H} \mathbf{R}_{x x^{-1}} \mathbf{A}\left(\mathbf{A}^{H} \mathbf{R}_{x x^{-1}} \mathbf{A}\right)
$$

That is, $\hat{\mathbf{f}}$ is the eigenvector corresponding to the minimum eigenvalue of $\mathbf{Q}_{s}$. Substituting $\hat{\mathbf{f}}$ into (30) yields the E-MCMV weight vector. 
Finally, the additional computational complexity of the enhanced LCMV-GAM and MCMV beamformers is considered. Performing the eigen-decomposition to obtain the signal subspace generally requires a computational load of $O\left(M^{3}\right)$, so the E-LCMVGAM and E-MCMV beamformers need more $O\left(M^{3}\right)$ computations than the LCMVGAM and MCMV beamformers.

\section{Numerical Examples}

Computer simulations are presented to evaluate and contrast the beamforming performance of the proposed approaches for coherent signal reception. We consider a uniformly linear array with 16 sensors, which corresponds to $M=16$, and the inter-element spacing is equal to the half of the wavelength of the signals. There are four signals impinging on the array from angles of $-40^{\circ},-20^{\circ}, 10^{\circ}$ and $30^{\circ}$, off the array broadside. The first three signals are assumed to be the desired coherent signals with different gains and the fourth signal is an uncorrelated interference with INR of $60 \mathrm{~dB}$. The desired signal is assumed to be of the form $s_{d}(t)=e^{j\left(2 \pi t / 3+\phi_{d}\right)}$, and the interference signal is $s_{i}(t)=e^{j\left(2 \pi t / 7+\phi_{i}\right)}$, where $\phi_{d}$ and $\phi_{i}$ denote the initial phase of the desired signal and interference respectively. Since $\mathbf{R}_{\mathrm{xx}}$ is not available in practical applications, it should be estimated from a finite sample of the received signals. Unless stated otherwise, the estimate of $\mathbf{R}_{\mathrm{xx}}$ is based on 1000 data snapshots and the DOAs of the coherent signals are assumed to be free of errors.

Example 1: Figure 1 shows the beam patterns of the MMSE, LCMV-GAM, MCMV, E-LCMV-GAM and E-MCMV beamformers when $\alpha_{1}=0.5, \alpha_{2}=0.63$ $\alpha_{3}=0.8$ and the coherent signals have SNRs of $-12 \mathrm{~dB},-10 \mathrm{~dB}$, and $-8 \mathrm{~dB}$, respectively. We see that all of these methods produce three main beams, with gains proportional to the amplitude of each coherent signal, for simultaneous reception of the desired signals and a null for successful suppression of the interference. Hence, they are capable in operating in the coherent signal environment. The performance of the E-LCMV-GAM, E-MCMV and MMSE algorithms is nearly identical, indicating the optimality of the eigenspace-based beamforming approaches. 


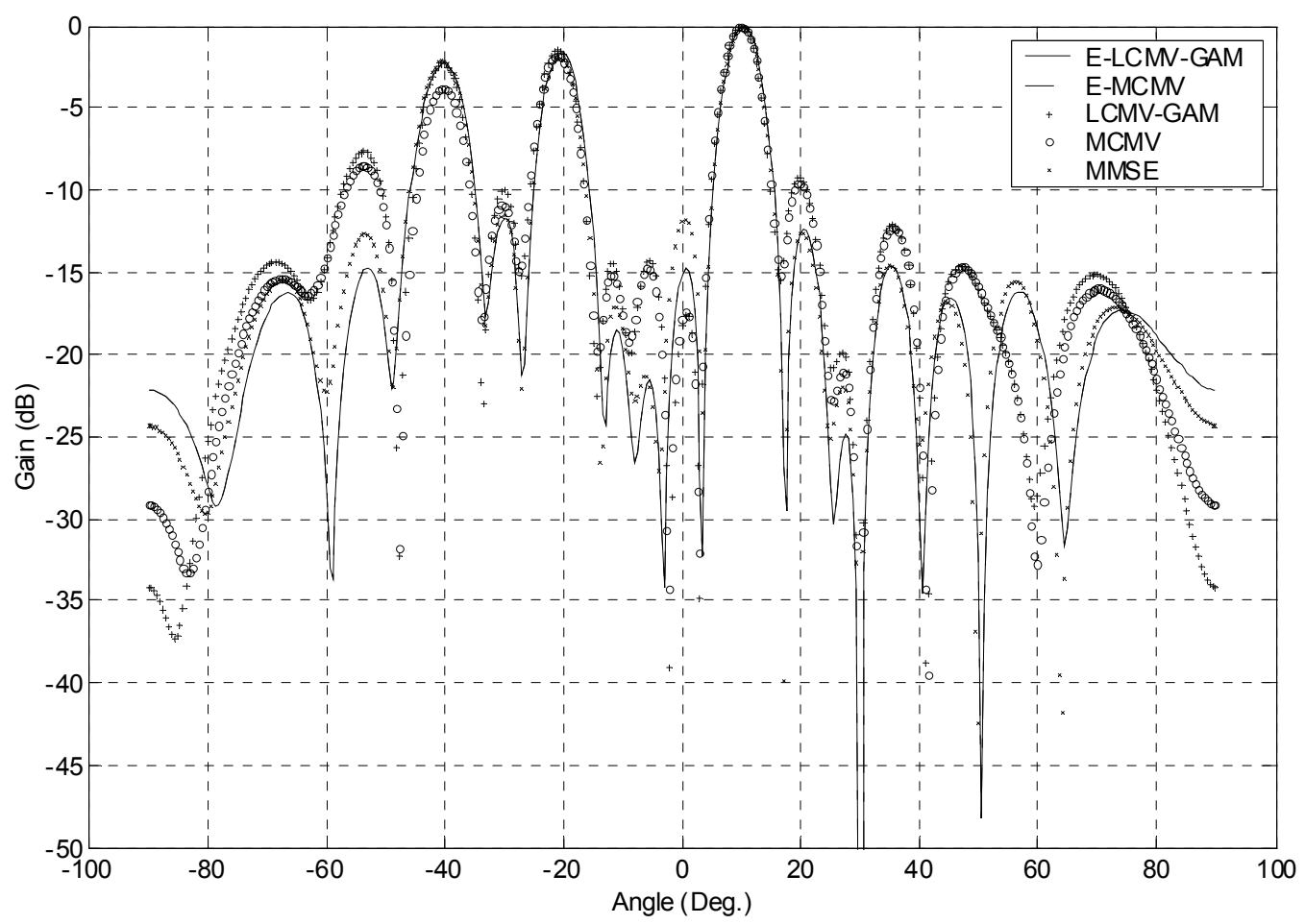

Figure 1. Beam pattern at low SNR

Example 2: The above test is repeated for a high SNR when $\alpha_{1}=0.5, \alpha_{2}=0.71$, $\alpha_{3}=0.89$ and the coherent signals have SNRs of 20,23 , and $25 \mathrm{~dB}$, respectively. The results are shown in Figure 2. It can be seen that the performance of the LCMV-GAM and MCMV beamformers suffers from significant degradation due to the more severe signal cancellation resulting from the use of the sample covariance matrix. On the other hand, the E-LCMV-GAM and E-MCMV algorithms successfully preserve the desired signals and the performance is almost identical with that of the MMSE approach. 


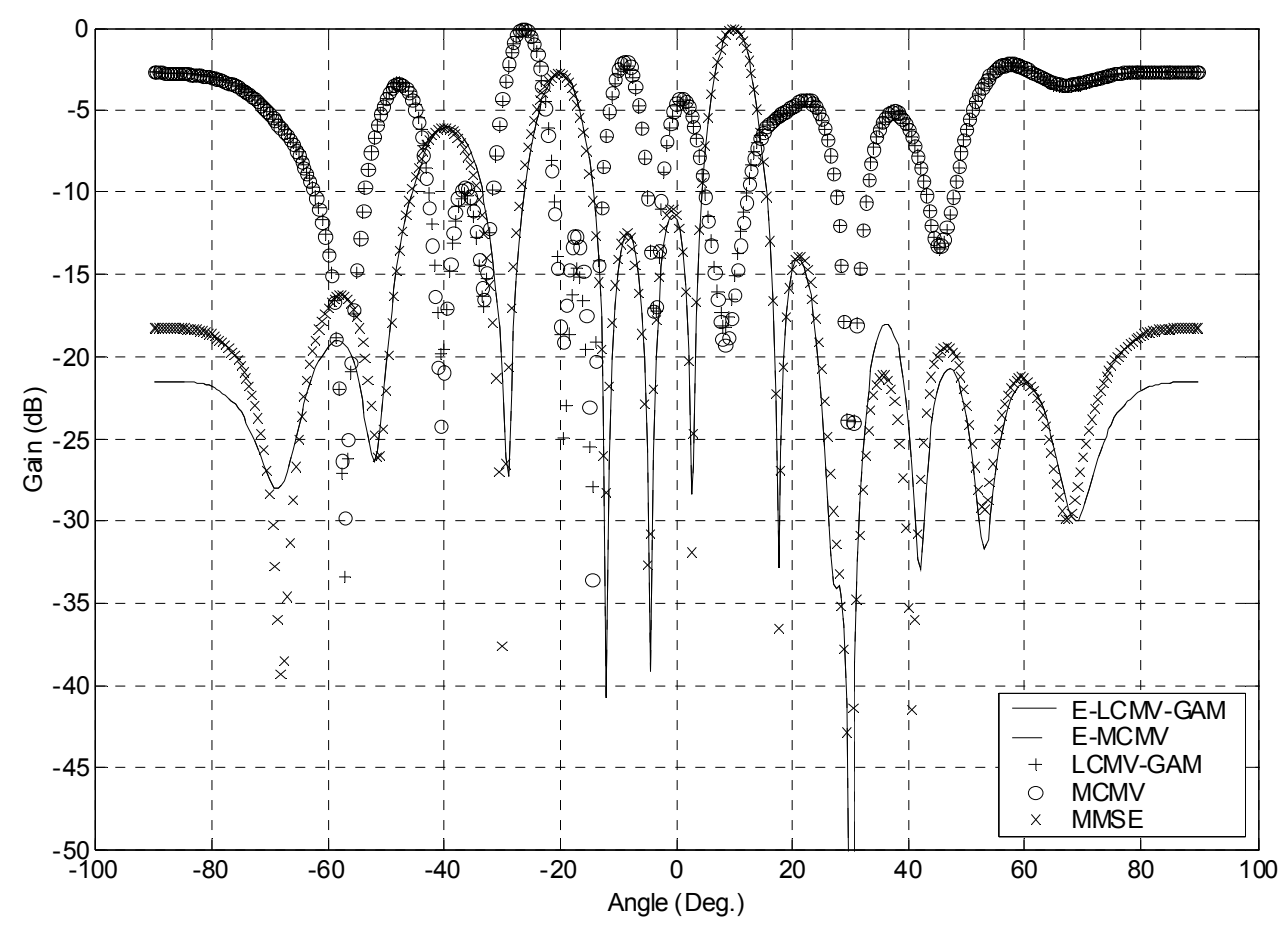

Figure 2. Beam pattern at high SNR

Example 3: The above test is repeated to examine the sensitivity of the output SINR to variations of phase angles of $\left(\alpha_{1}, \alpha_{2}, \alpha_{3}\right)$ while maintaining same magnitudes as Example 2. The phase angles of $\left(\alpha_{1}, \alpha_{2}, \alpha_{3}\right)$ are chosen as $(-\phi, 0, \phi)$, where $\phi$ is changed from $-180^{\circ}$ to $180^{\circ}$. The results are shown in Figure 3. We see that all these methods are sensitive to variations of phase angles, and the performance of the E-LCMV-GAM and E-MCMV algorithms is almost identical with that of the MMSE approach. 


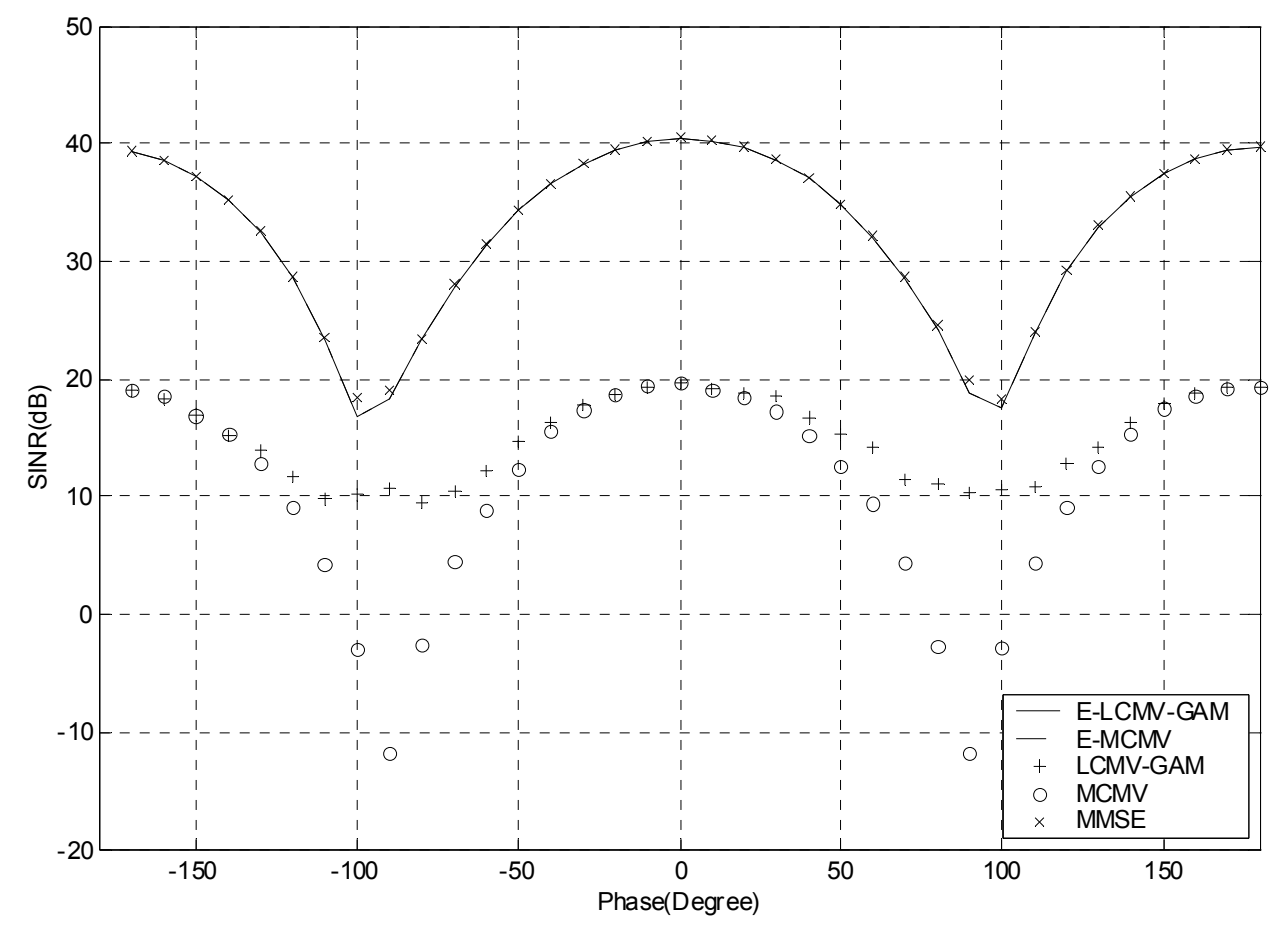

Figure 3. Output SINR to variations of phase angles of $\left(\alpha_{1}, \alpha_{2}, \alpha_{3}\right)$

Example 4: The fourth experiment evaluates the output SINR of the five beamformers as a function of the input SNR. For simplicity, the SNRs of the three coherent signals are chosen to be identical. The results are plotted in Figure 4 and we perform 50 Monte Carlo trials for each SNR point. When the SNR is moderate, that is from $-20 \mathrm{~dB}$ to $-5 \mathrm{~dB}$, the performance of the four proposed approaches is close to that of the MMSE beamformer. On the other hand, when the SNR is higher, the performance of the LCMV-GAM and MCMV methods degrades due to the signal cancellation resulted from using finite samples, while the performance of the E-LCMV-GAM and E-MCMV approaches is again close to the optimum MMSE algorithm. Furthermore, we see that the LCMV-GAM beamformer is slightly superior to the MCMV method at very low SNR conditions, which is consistent with our analysis in Section IV. It is also noted that at very low SNRs, the performance of the E-LCMV-GAM and E-MCMV methods is not optimum, because of the incorrect subspace decomposition in such conditions. 


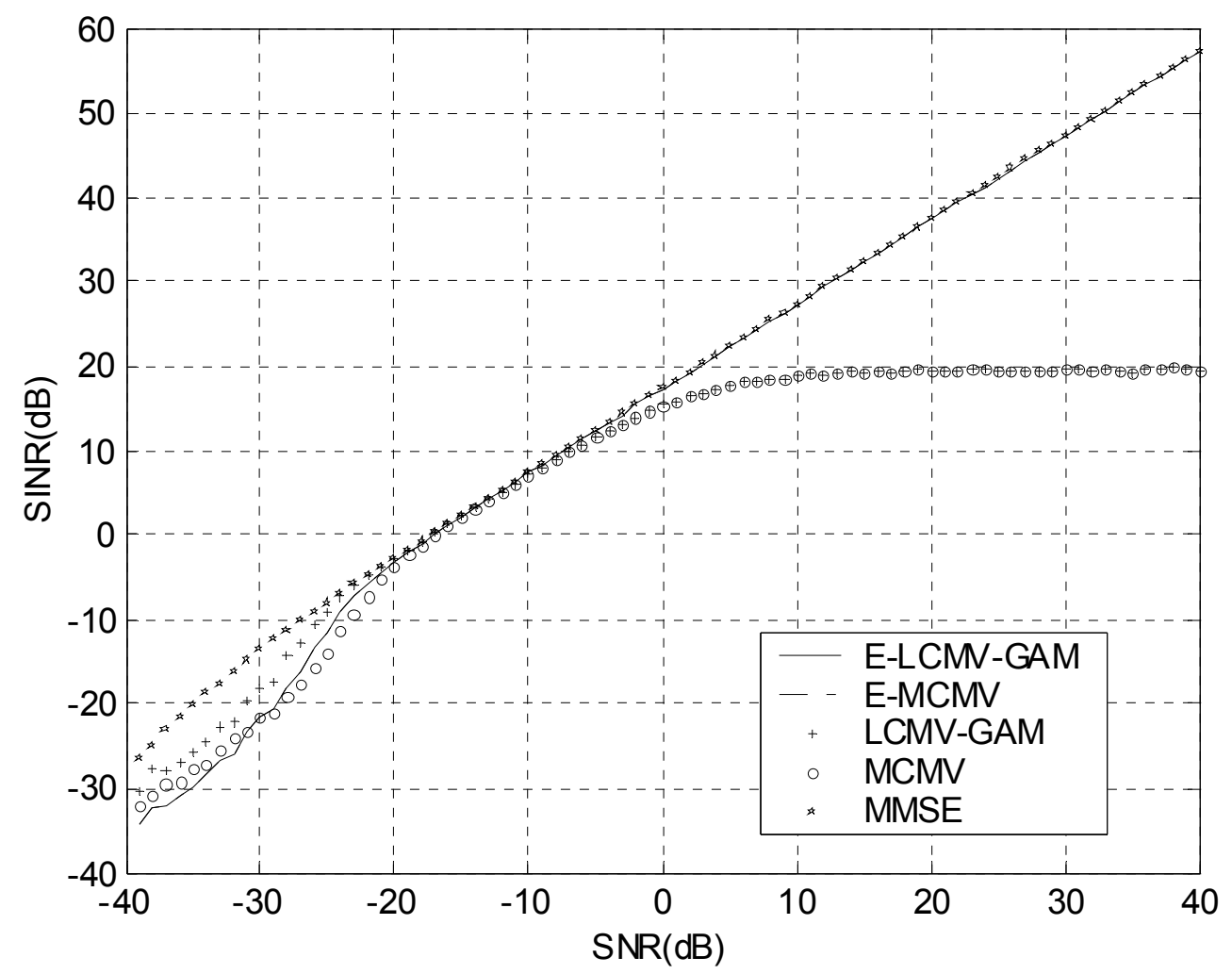

Figure 4: Output SINR versus SNR

Example 5: This test investigates the convergence behavior of the five beamformers. Figure 5 shows the average output SINR versus the number of snapshots, where each data point is based on 50 Monte Carlo trials. The SNR and the complex amplitudes of the coherent signals are the same as those in Example 2. We observe that the E-LCMV-GAM and E-MCMV methods converge much faster than the LCMV-GAM and MCMV algorithms, and they achieve maximum SINR with a small sample size. 




Figure 5: Convergence behavior of the beamformers

Example 6: This experiment investigates the performance of the five methods in the presence of DOA estimation errors and the results are plotted in Figure 6. The SNR and the complex amplitudes of the coherent signals are identical to those in Example 1. We see that the LCMV-GAM and MCMV approaches suffer significant degradation in performance due to the severe signal cancellation, whereas the projection-based ELCMV-GAM and E-MCMV methods are more robust to steering errors and achieve higher SINRs than those of the LCMV-GAM and MCMV beamformers, which approach MMSE performance. 


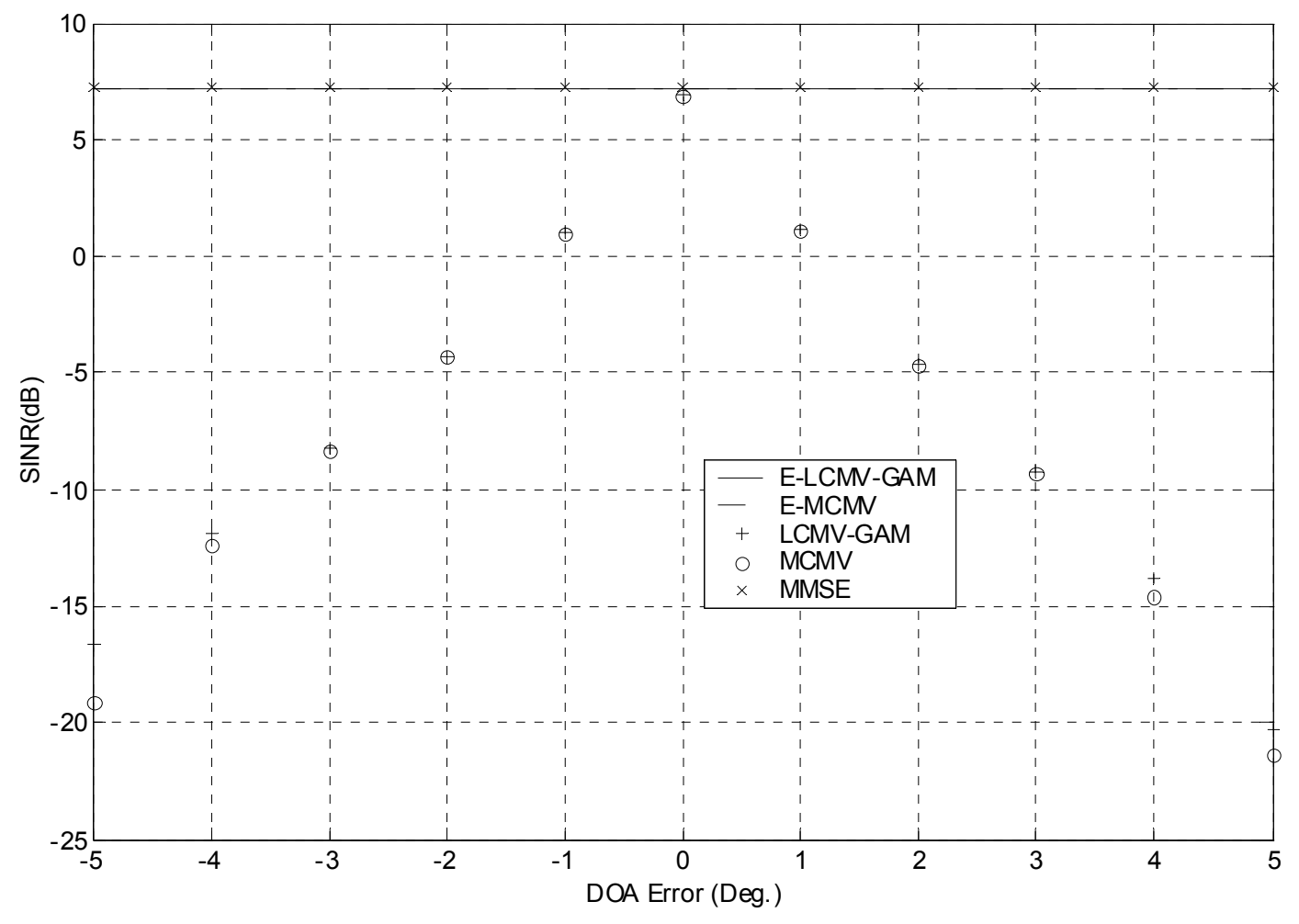

Figure 6: Output SINR versus DOA error

Example 7: This experiment examines the sensitivity of the performance due to variations of array length of the five methods and the results are plotted in Figure 7. The SNR and the complex amplitudes of the coherent signals are identical to those in Example 1. We see that the performance of all the methods generally improves as the array length increases. 


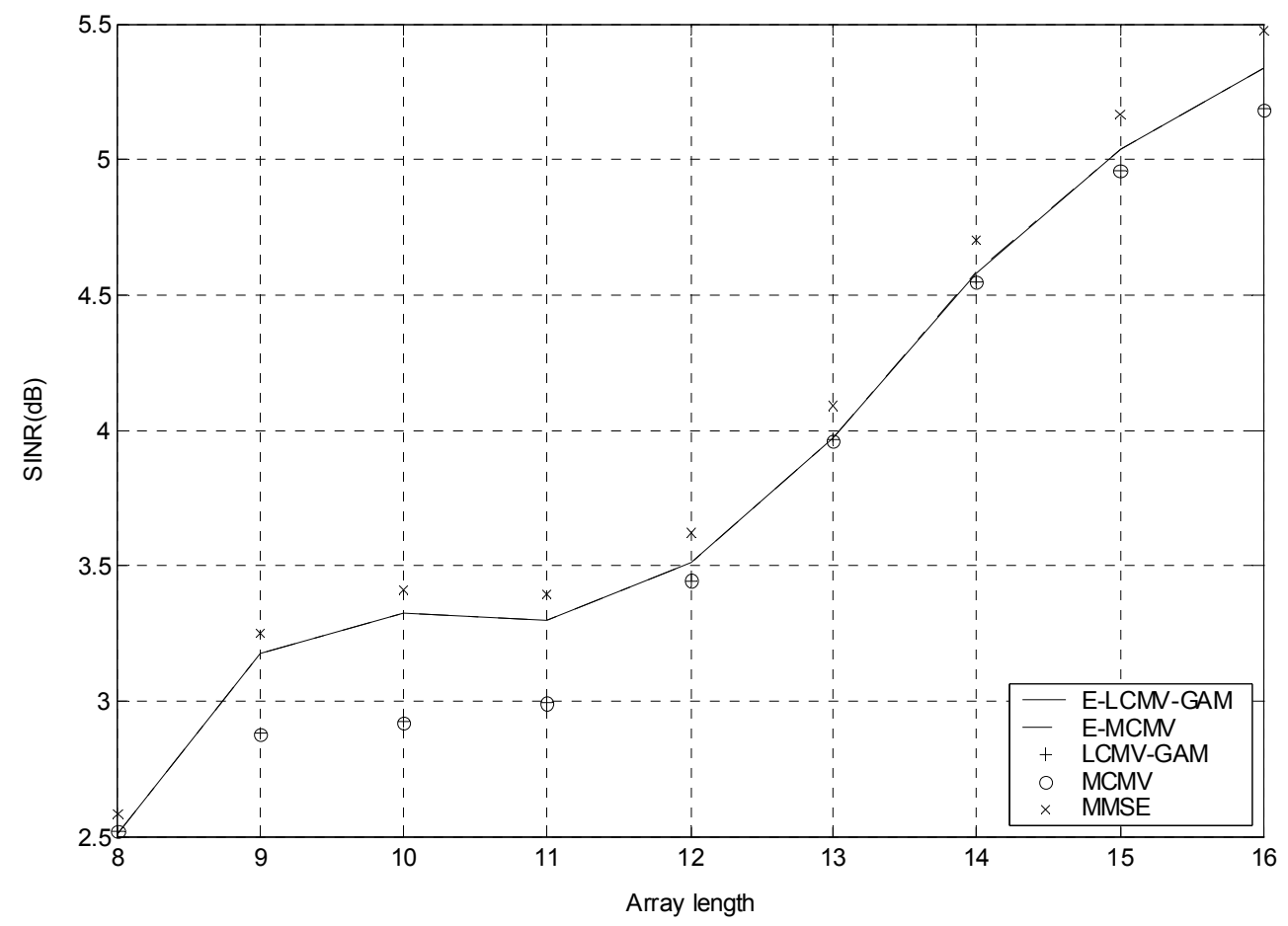

Figure 7: Output SINR versus Array length

Example 8: The last experiment investigates how the occurrence of a singlecoherent jammer would affect the adapted array pattern and the results are plotted in Figure 8. The SNR, INR and the complex amplitudes of the coherent signals are identical to those in Example 1, except the interference at $30^{\circ}$ is a single-coherent jammer. We see that correlation between desired signal and unwanted interferences can make the adaptive beamformers not only fail to form nulls in the direction of the coherent interferences, but also tend to cancel the desired signal in the output. For coping with the performance degradation due to the presence of coherent jammers, our approaches can be used in conjunction with spatial smoothing technique [1] or Toepliz approximation approach [10, $6,7]$ to alleviate the coherent jammer effect. 


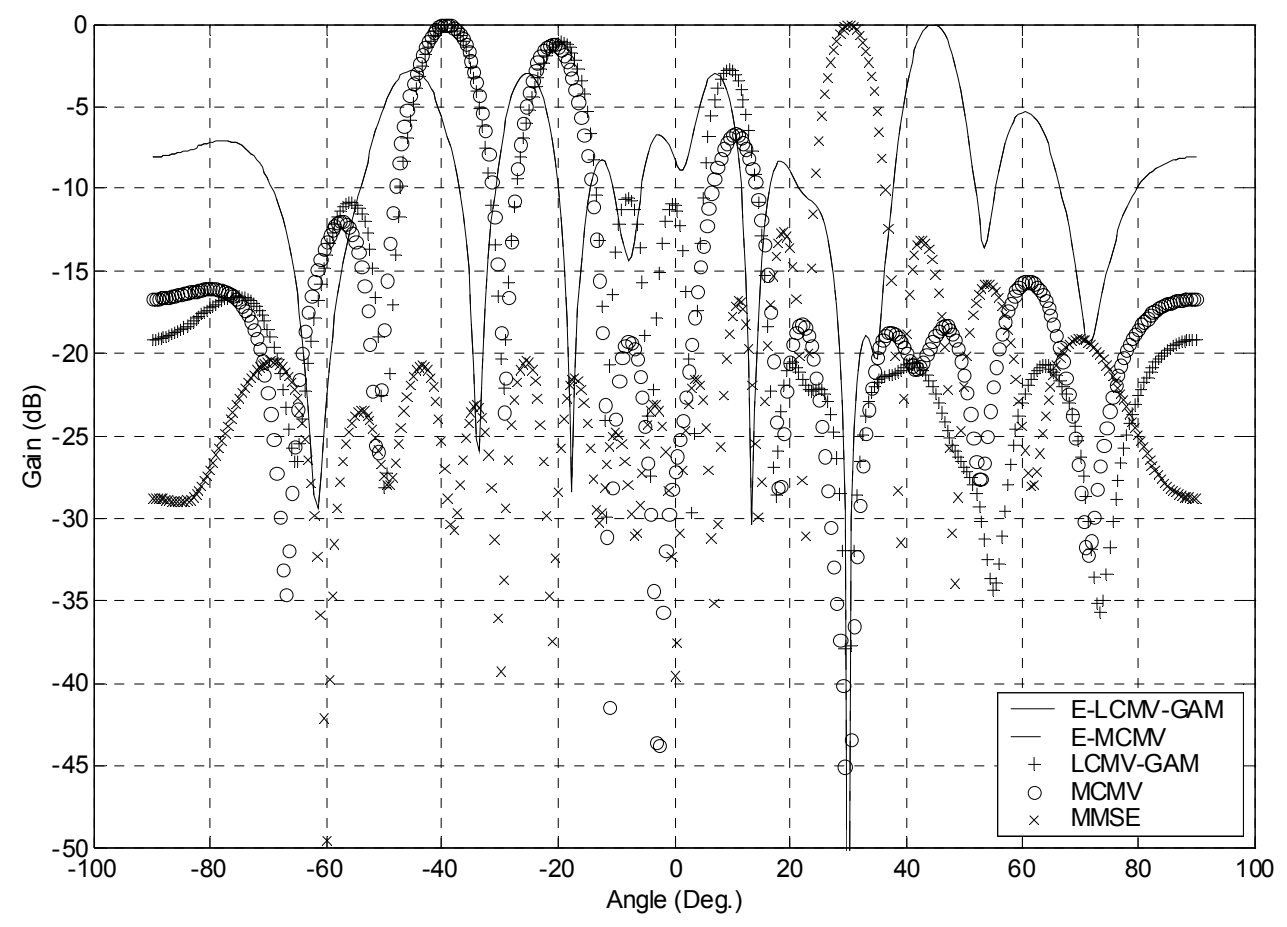

Figure 8. Beam pattern for signal-coherent jammer

\section{Conclusions}

Two novel beamforming approaches for coherent signal reception in the presence of uncorrelated interferences, assuming that the direction-of-arrival (DOA) information of the coherent signals is known, have been developed. The first approach performs linearly constrained minimum variance (LCMV) beamforming together with estimation of the complex amplitudes of the coherent signals. While the second approach is based on a multiple constrained minimum variance (MCMV) criterion, where we make use of the DOAs of the coherent signals to construct the constraints such that all coherent signals are preserved. Basically, the idea of these two approaches is to optimally combine all coherent signals to yield the maximum output signal-to-interference-plus-noise ratio. The performance of the LCMV and MCMV beamformers is analyzed and their improved versions via utilization of signal subspace projection are also suggested.

Numerical examples illustrate that the performance of the proposed beamformers is quite reliable, in particular, the two improved versions performs almost identically with 
the minimum mean square error (MMSE) beamformer, except at very low signal-to-noise ratio conditions. Although the MMSE approach is optimum for all cases, it requires training signals, while our approaches are blind. Nevertheless, our algorithms need the DOA information of all coherent signals as well as the number of signal and interference sources. Furthermore, the proposed methods in their current forms apply to non-coherent jammers only. Development of beamformers for coherent signals in more general data models will be one of our future research directions.

\section{Acknowledgement}

The work described in this paper was supported by a grant from City University of Hong Kong (Project No. 7001203).

\section{REFERENCES}

[1] V. U. Reddy, A. Paulraj and T. Kailath, "Performance analysis of the optimum beamformer in the presence of correlated sources and its behavior under spatial smoothing," IEEE Trans. Acoust., Speech, Signal Processing, ASSP-35 (July 1987) 927-936.

[2] Y. Bresler, V. U. Reddy, and T. Kailath, "Optimum beamforming for coherent signal and interferences," IEEE Trans. Acoust., Speech, Signal Processing, ASSP-36 (June 1988) 833-843.

[3] Y. L. Su, T. J. Shan and B. Widrow, "Parallel spatial processing: A cure for signal cancellation in adaptive arrays," IEEE Trans. Antennas Propag., AP-34 (Mar. 1986) 945-947.

[4] J.F. Yang and M. Kaveh, "Coherent signal-subspace transformation beamforming," Proc. IEEE 137 (Aug. 1990) 267-275.

[5] C.C. Yeh and W.D. Wang, "Coherent interference suppression by an antenna array of arbitrary geometry," IEEE Trans. Antennas Propag., AP-37 (Oct. 1989) 1317-1322.

[6] L. C. Godara, "Beamforming in presence of correlated arrivals using structured correlation matrix," IEEE Trans. Acoust. Speech Signal Process., Vol.38, January 1990, pp.1-15.

[7] M Hatem El-Ayadi, Emad K. Al-Hussaini, Eid A. El-Hakeim, "A combined redundancy averaging signal enhancement algorithm for adaptive beamforming in the presence of coherent signals and interferences", Signal Processing, Vol.55, 1996, pp.285-293.

[8] E. Gonen and J. M. Mendel, "Applications of cumulants to array processing_Part III: blind beamforming for coherent signals," IEEE Trans. Signal Processing, SP-45 (Sep. 1997) 2252-2264.

[9] T.T. Lin, "A novel beamformer with multipath signal reception," Proceedings of the IEEE National Aerospace and Electronics Conference, 2000. pp.638 -644.

[10] J.H. Lee and T.F. Hsu, "Adaptive beamforming with multiple-beam constraints in the presence of coherent jammers," Signal Processing, Volume 80, Issue 11, November 2000, pp.2475-2480. 
[11] T.S. Lee and T.T. Lin, "Reception of coherent signals with steering vector restoral beamformer", Signal Processing, Volume 72, Issue 3, February 1999, pp.141-145.

[12] Y.T. Lee; J.H. Lee; "Direction-finding methods for cyclostationary signals in the presence of coherent sources", IEEE Trans. Antennas Propag., AP-49 (Dec 2001) 1821 -1826.

[13] M. Zoltowski and F. Haber, "A vector space approach to direction finding in a coherent multipath environment”, IEEE Trans. Antennas Propag., AP-34 (Sep. 1986) 1069 -1079.

[14] E. Gonen, J.M. Mendel and M.C. Dogan, “Applications of cumulants to array processing. IV. Direction finding in coherent signals case” IEEE Trans. Signal Processing, SP-45 (Sep. 1997) 2265 -2276.

[15] W. Du and R.L. Kirlin, "Improved spatial smoothing techniques for DOA estimation of coherent signals", IEEE Trans. Signal Processing, SP-39 (May 1991) 1208 -1210.

[16] M. Honig, U. Madhow and S. Verdu, "Blind adaptive multiuser detection,” IEEE Trans. Inform. Theory, IT41 (July 1995) 944-960.

[17] M.K.Tsatsanis and Z.Xu, "Performance analysis of minimum variance CDMA receivers," IEEE Trans. Signal Processing, SP-46 (Nov. 1998) 3014-3022.

[18] L. Chang and C.C. Yeh, "Performance of DMI and eigenspace-based beamformers", IEEE Trans. Antennas Propag., AP-40 (Nov 1992) $1336-1347$.

[19] D.D. Feldman and L.J. Griffiths, “A projection approach for robust adaptive beamforming”, IEEE Trans. Signal Processing, SP-42 (Apr 1994) $867-876$.

[20] J.L. Yu and C.C. Yeh, "Generalized eigenspace-based beamformers", IEEE Trans. Signal Processing, SP-43 (Nov 1995) $2453-2461$. 